\title{
The Challenge of IPP's Plant Characteristics against the Power System Security
}

\author{
A. C.S. Wijayatilake
}

\begin{abstract}
When a utility owned new power plant is constructed it is a traditional practice to carry out detailed power system analysis to identify the most appropriate combination of plant characteristics to be incorporated into the new power plant since it has to be operated with the rest of the equipment used in the transmission system complying with the operational policies and standards. Hence, plant features and operational characteristics such as generator parameters, controller settings, excitation, relay settings, power factor and other auxiliary equipment settings etc. are determined based on the outcome of the detailed power system analysis done by transmission network planners. When system planners determine that a power plant feature is needed it is provided.
\end{abstract}

However, now we are in a competitive environment and Independent Power Producers (IPPs) more concentrated about their return on selling power neglecting the importance of coordinated system design and operation. Cost optimization results in removing or limiting certain valuable features from the IPP owned power plant which are very important for system operators to maintain the expected level of system security. At the prevailing environment utility has very limited control over determining the characteristics of IPP's machines. Resulting plant characteristics are far from optimum from a system standpoint. As a consequence the transmission network security reduces and increases the probability of having system blackouts. If there is a lack of long range planning these issues may not highlight until the operational staff realize that IPPs may not provide traditional plant characteristics and features when system is subjected to the severe stress. However, then the utility has to introduce more costly transmission solutions to regain the network security.

This paper addresses the technical issues behind this problem.

\section{Introduction}

The present electrification level of Sri Lanka is $78 \%$ and the annual demand for the electricity is increasing every year by $8 \%$ [1]. It is the duty of the relevant authorities to increase the generation capacity to cope with this high demand growth. Since the available hydro potential for generating electricity has been fully utilized by now Sri Lanka has to depend on thermal power generation in the future. During some drought periods in year 2001 more than $70 \%$ of the daily generation was produced using thermal plants [2].

Up to 1996, Ceylon Electricity Board (CEB) had the monopoly in electricity generation and it was the responsibility of CEB to prepare the annual generation plan, which describes the type, place and size of the oncoming generators through detailed economic analysis. Thus, in the past, CEB had a direct involvement in various activities of power plant connecting processes such as planning, preliminary designing and feasibility studies etc. Utility engineers directly involved in these activities with their views and experience on power system operation and control were an advantage for consultants to design the most suitable plant to match with needs of the local grid. At a final stage in construction utility engineers participated in commissioning activities and thereby CEB had an opportunity to foresee and rectify any problems, which would affect adversely on system performance once the machine is operated synchronizing with the grid.

However, this situation has been changed drastically due to the government decision to allow Private Power Producers (IPP) to enter the local electricity industry. It has been decided that in the future private sector should carry out all new power generation projects and the

Eng. A. C. S. Wijayatilake, B.Sc(Eng), Mphill, MIESL, MIEE, Presently, System Planning Engineer, Ceylon Electricity Board. 
government decided to limit government funds on power generating facility development [3].

Once, this policy was changed, the commercial aspects of power generation were given high priority over the related technical aspects. IPP always looks for possibilities of getting a high return for their investments. Hence, their willingness to generate maximum possible power with minimum cost and thereby earn a large profit. To achieve their target, IPPs try to cut down many traditional features incorporated to utility owned generators, which are not essential merely for power generation, but to enhance the power system security and safeguard the system from blackouts and severe disturbances. As a result, IPPs reduces the system reliability and utility has to do a massive investment on transmission network reinforcement to compensate the adverse effects introduced by IPP plants and to improve the reliability level of the network. However, some adverse effects introduced by IPPs cannot be fully offset. The objective of this article is to highlight the important technical issues behind the grid connection of IPPs generators.

The discussion is focused on different important subtopics as following though all relevant issues are very much interrelated to each other.

\section{Issues related to the voltage control of the network and voltage stability}

Voltage at a power system is allowed to vary within a very narrow range since the larger deviations adversely effect on the network performance as well as lifetime of the equipment connected. At steady state transmission system voltage is maintained within of the rated value [1]. Maintaining the right voltage profile is therefore the responsibility of the system operator.

Voltage is a local variable in a power system and it is a function of the reactive power flow at the particular point. Mathematically the relationship can be expressed as

$$
\text { Voltage }(k V)=f\left(V A r_{\text {generated }}-V A r_{\text {load }}-V A r_{\text {losses }}\right)
$$

where the VAr losses can either be positive or negative depending on the network loading. For instance, in Sri Lankan transmission network at day time peak load more equipment consume reactive power and the VAr losses are negative. But at minimum load occurring at mid night is mainly resistive and VAr demand is less than the VAr generated by the transmission line capacitance. Thus VAr losses are positive. The consequences of failing to achieve the VAr balance can range from excessively low voltage and increased MW losses under heavy load conditions to excessively high voltage under light load conditions. Both conditions may result in damage to the plant and equipment connected and seriously reducing the system security.

It is very clear that reactive power management is essential in a transmission system to control the voltage profile and improve the system security. Reactive power sources like capacitor banks are located at load centers to minimize the $\mathrm{VAr}$ flow and reduce the loading on transmission facilities. Though, switched shunt capacitors are very economical and hence extensively used in the power systems, they are suitable to maintain the reactive power balance at steady state or at instances where system voltage is dropping at a slow rate. On the other hand reactive power generated by capacitors will reduce dramatically when the network voltage is reducing and as a consequence capacitors fail to provide the necessary support at the most required instances by making the situation worse. The fast acting reactive power resources should be located at identified places in order to provide the reactive power requirement when rapid network changes are taking place i.e. sudden tripping of a large generator, heavily loaded line or sudden switching of large active/reactive power loads, cascaded tripping of equipment due to system faults etc. At a severe disturbance, if the reactive power sources fail to bring the system voltage back to the allowable limit within a limited period, the fast decreasing voltage causes a system failure by operating under voltage relays of generator auxiliaries and subsequent tripping of generators connected to the system. At similar situations network may collapse due to voltage instability. Therefore it is a practice in system operation to have more than the one third of the reactive power support kept available at dynamic sources such as generators, SVCs or synchronous condensers [4].

Power system planners are very much vigilant in overcoming voltage instability situations 
when system security is concerned and planned to locate dynamic reactive power resources such as SVCs or generators having fast reactive power capability at several suitable places of the network. General operational practice is to use static capacitors as much as possible at the steady state while maintaining the generator reactive power as a spinning reserve to be used in system disturbances to avoid system leading to the voltage instability. Thus, the reactive power capabilities of the generators are very important for the system security point of view. Hence when a new generator is ordered by a utility the transmission planners are mainly concerned about the ability of the generator to provide maximum possible reactive power and opportunities available for enhancing the reactive power capability by manipulating the relevant settings of controllers, limiters and generator protective relays favourable to the system operations.

Reactive power capability of a generator can be expressed with the help of a typical capability curve, as shown in Fig.1, provided by the generator manufacturer. Generator active power availability is dependent on the prime mover selected. The ability of a synchronous generator to generate reactive power is restricted by the increases in temperature in the generator windings. As indicated in the Fig.l, capability curve limits can be further expanded if the gas pressure of the generator cooling system can be increased in order to maintain the synchronous generator temperature below the electrical insulation temperature class limit described by

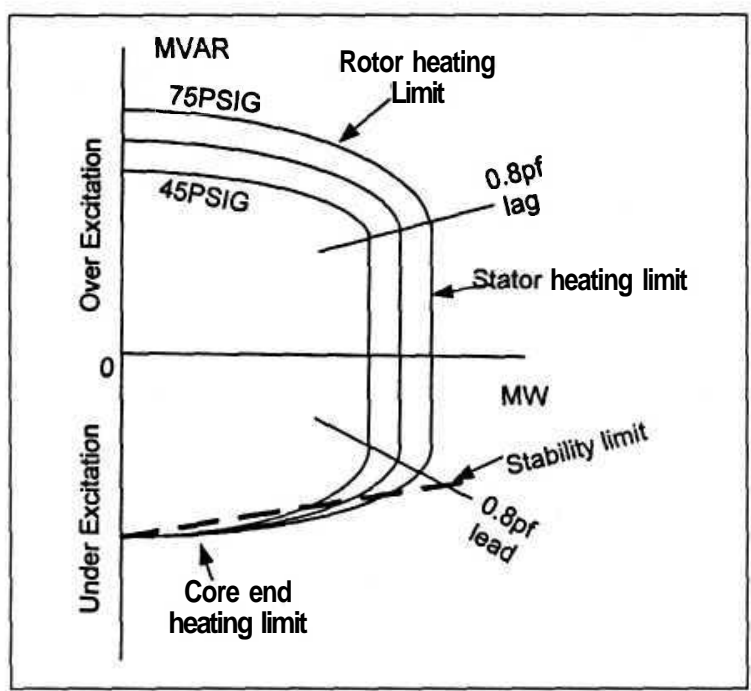

Fig.1: Typical capability curve of a synchronous generator the relevant standards. The figure shows that when the pressure of the generator cooling gas (normally Hydrogen) increases the upper boundary of the capability curve further expands which represents the enhancement of the thermal capability of the rotor field. Approximately the upper boundary of the capability curve is an arc with a center at a value equal to the short circuit ratio (SCR) in per unit on the $\mathrm{VAr}$ axis and a radius of $E / X$ where $E_{f}$ is the field excitation voltage given that the synchronous generator terminal voltage is one per unit. The right hand boundary is the synchronous generator stator current limit. The center of the arc defining this limit is the origin. The lower boundary is the end iron heating limits, which occur when the generator is operated at under excitation limits to absorb VAr. As shown in Fig.l the intersection point of the stator and field thermal limit determines the power factor and rated capacity of the generator.

Suppose a new generator is to be connected to the system. It is assumed that the prime mover rating is $1 \mathrm{pu}$. If this machine is ordered by IPP they look for the feasible cost reduction measures and are mainly concerned on getting maximum possible active power with minimum cost. Reactive power capability is not a matter for them since there is no payment scheme for IPP's reactive power generation. Hence IPP prefers to select a machine, which has a very high rated power factor. When generator's power factor is high it's reactive power generation is low and as a result the current flowing through the windings are low. Thereby conductors with smaller cross section can be used in windings and the cost of the windings can be reduced significantly. In addition to that the cost of the cooling system can be further reduced due to the reduced thermal losses in the windings. Similarly the other trend in low cost generator design is to reduce the Short Circuit Ratio (SCR). However, reducing SCR to very low values results in increasing the winding cost. Therefore SCR is optimized to have minimum possible value, which can be justified in terms of the machine manufacturing cost. The VAr absorbing capability of the generator is proportional to the SCR. IPPs always prefer to have low SCR machines since they are not willing to operate their machines at under excitation mode to absorb VAr at light load or 
emergency situations where system has excess VAr. Low SCR is achieved by reducing the machine air gap. It will reduce the machine inductance and as a consequence less current is drawn to the winding resulting in low losses with consequent savings in machine mmf, size, weight and cost [5]. However if the machine is ordered by the utility the utility planning unit is more concerned about the maximum reactive power generating and absorbing capability of the generator to improve the system security. Definitely the utility will order a generator with a low rated power factor to increase VAr generating capability and the higher SCR to increase the under excitation limit operation of the generator at emergencies. This situation is clearly presented in Fig.2 where (A) for a generator with 0.8 power factor with high SCR and (B) for a generator with 0.95 power factor and low SCR. The (B) is the IPP selection due to the low cost but (A) is the utility selection due to the flexibility in reactive power control. It is assumed that the prime mover rating is $1 \mathrm{pu}$ for both cases. The figure shows that the machine (A) having $18 \%$ larger MVA rating than (B). However, the reactive power capability of (A) is $225 \%$ larger than (B) with a little more investment. Since SCR is smaller for the generator (B) its $\mathrm{VAr}$ absorbing capability is very much lower than the generator $(\mathrm{A})$.

This situation can be easily realized when IPP generators presently operating in the network are compared with utility owned machines. Always-IPP generators are having higher power factor ratings above 0.95 and power factor of the utility ordered generator is typically 0.8 . Therefore IPP machine will not provide the

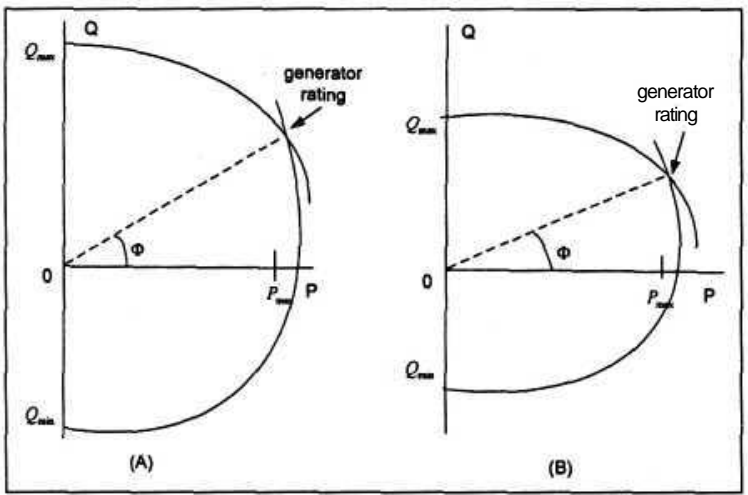

Fig.2: Reactive power capability of two different generators connected with same size turbine (A) generator having rated power factor $=0.8$ and high SCR (B) generator having rated power factor - 0.95 and low SCR expected reactive power support and system operators have to switch on fast acting alternative reactive power sources such as SVCs to face for the emergency system disturbances. Otherwise degrading of the network security by low cost IPP machines cannot be fully offset.

Even though capacitors are economical reactive power sources, the generators have a specialty. That is because of the generator supplied reactive power can be increased more than the rated values for a short period to face the critical system disturbances leading to the system collapse due to voltage instability [4]. According to the ANSI C50.13-1977, presented in Fig.3, stator and rotor current carrying capabilities can be overloaded between the range approximately $200 \%$ for 10 seconds and $110 \%$ for 2 minutes [6] The transmission planners are more concerned about this very important capability of the generator. That is because during the severe disturbance the generator can tolerate the over loading caused due to the rapid VAr inbalance in the system for a short period. That small time period is sufficient for automatic operation of the protection devices or connect/disconnect the equipment necessary to bring the network back to the normal situation. At the same time the spinning reserve will activate and operators will reschedule the generation to normalize the system. However the generator over loading capability will depend on the generator cooling system. The utility is always ready to spend additional expenditure for cooling system development to get the short-term overload capacity of their generator. However, for IPP it is an immaterial factor and they do not allow overload operation of their machine for any reason to avoid the possible life time reduction of generator windings due to high thermal stresses and to minimize the maintenance cost.

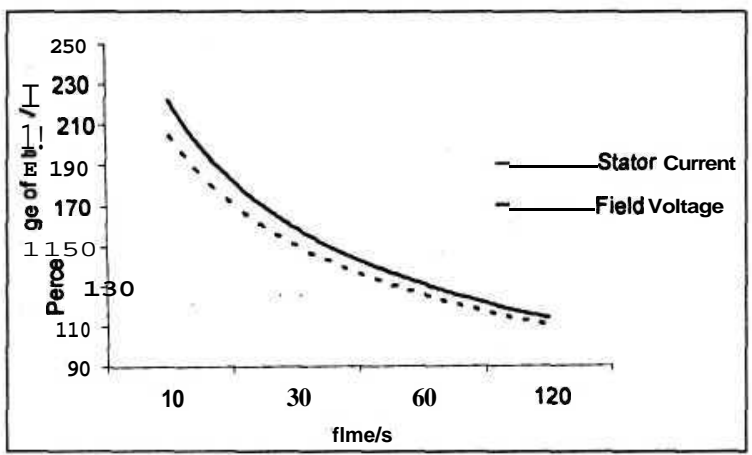

Fig.3: Generator stator and field overload capabilities as given in ANSI C50.13-1977 


\section{Issues behind the limiter settings and plant controller tuning}

IPPs want to minimize their operation cost and prefer to operate their machine at automatic mode rather than frequent intervention of the operator. Therefore the machines operating at extreme operating conditions are avoided by adopting system automation techniques. It is common to introduce limiters on control loops aiming machines not operating at very critical operating points. Similarly limiters are introduced into machine excitation system as shown in Fig.4 and their settings are adjusted by considering both the network and machine requirements [8]. When generator requirements are concerned these limiters will avoid the machine operating outside the limits defined in the capability curve provided by the manufacturer. Thereby windings are protected from high thermal stresses. As a consequence the lifetime of the generator windings are extended. However, if these limiters are not properly tuned to match with the network requirement, the generator may fail to provide the expected support at severe disturbances and may lead the network to blackout situation. The following discussion is on the importance of the tuning of the limiters at the excitation system shown in Fig.4.

To ensure the generators are not operating other than the rated operating points the IPPs manipulate the settings in Automatic Voltage

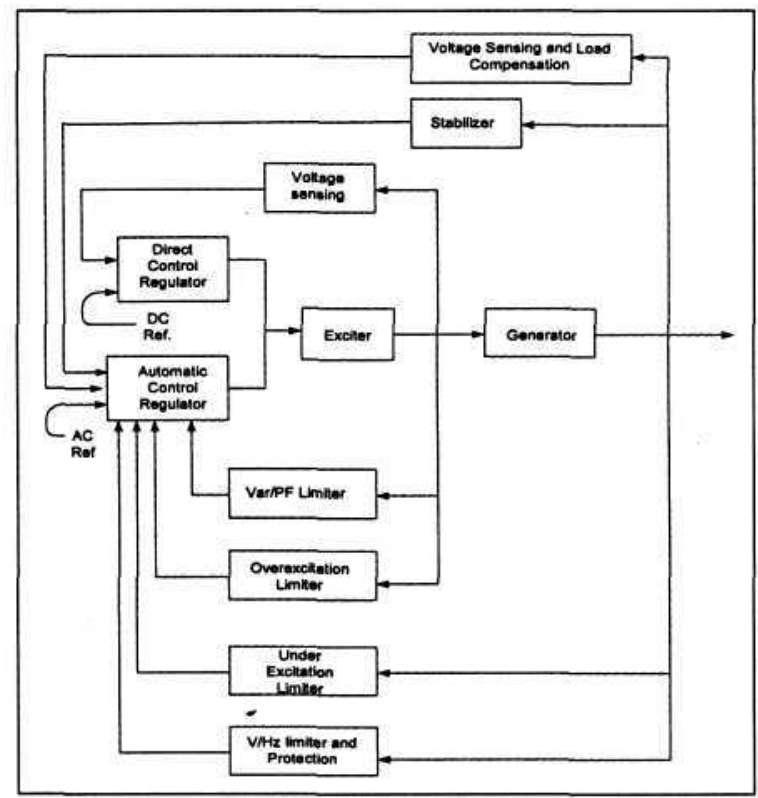

Fig.4: Components of Typical Excitation Control System
Regulator (AVR) systems more conservatively. For instance the over excitation limiter settings can be adjusted more conservatively to avoid overloading on the stator and rotor windings when system voltage is getting down due to a disturbance. At similar occasions the voltage of the network should be boosted up immediately by pumping Var to the network. To increase the Var production of the generator the generator excitation should be increased. The resulting high current passing through the stator and rotor windings may reach the thermal limit. The function of Over Excitation Limiter (OEL) is to limit the excitation current to a pre desired value in order to avoid exceeding the thermal limits of the windings. When the generator winding current reaches the maximum possible value an alarm is set to inform the operator and if the operator fails to change the controller from automatic mode to the manual mode the excitation is set at the rated value after an allowable time period. Thereafter the machine cannot control the system voltage any more. Then the generator behaves as a voltage following machine and looses the voltage controlling characteristics. OEL settings are initially set by the manufacturer considering the winding thermal limits to match with the capability curve and subsequent fine adjustment is necessary at the commissioning stage to match with the network operations. Generally the maximum over voltage allowed at busbars defined in grid code and transformer saturation limits defined in transformer standards are the main considerations. The typical settings are $110 \%$ of nominal bus voltage or $110 \%$ of nominal volt/Hz [7]. The idea is to protect the generator windings as well as auxiliaries connected to the generator busbar from over voltages at maximum Var generation of the machine. When a utility owned generator is commissioned these settings are manipulated according to the network conditions but it is common to see that IPPs want to keep the manufacturer's setting without further adjustment. This will affect adversary at some disturbances, which cause reactive power deficit in the network. On similar occasions unnecessary disconnection of generators due to the operation of over voltage relay of auxiliaries or $\mathrm{V} / \mathrm{Hz}$ protection relay of step up transformer is possible by further weakening the system. 
Other important setting is the Under Excitation Limiter (UEL) setting and it will come into play when the machine is absorbing VArs to reduce the higher system voltage. Similar Var absorption is noticed generally at light load condition or when the generator is connected to a long line or at a sudden tripping of a Var absorbing generator. Absorbing VArs will bring down the system voltage and the resulting low excitation can move the operating point towards the stator end heating limit or the generator stability limit marked at the operating chart presented in Fig.5. If machine is allowed to operate with low excitation there is a tendency for a generator to loose the synchronism and become unstable. Therefore under excitation limiter will bring the machine back to a pre determined excitation level after a defined period and if the UEL cannot take the control of AVR then machine is tripped by activating the Loss Of Excitation (LOE) relay. If a machine is unable to absorb the excess VAr and subsequent tripping of the unit by activating LOE relay from the system may cause the problem more severe and may lead to a total system collapse. The exact setting of LOE is vital for system stability is concerned and the ability of the machine to operate at extreme ends of the capability curve should be investigated at the commissioning stage. At commissioning the manufacturer settings of UEL and LOE should be adjusted to get the maximum benefit for the system at severe disturbances. Otherwise, high voltage damage to customers or utility equipment is possible and unnecessary tripping of the generator may lead to a total blackout [8].

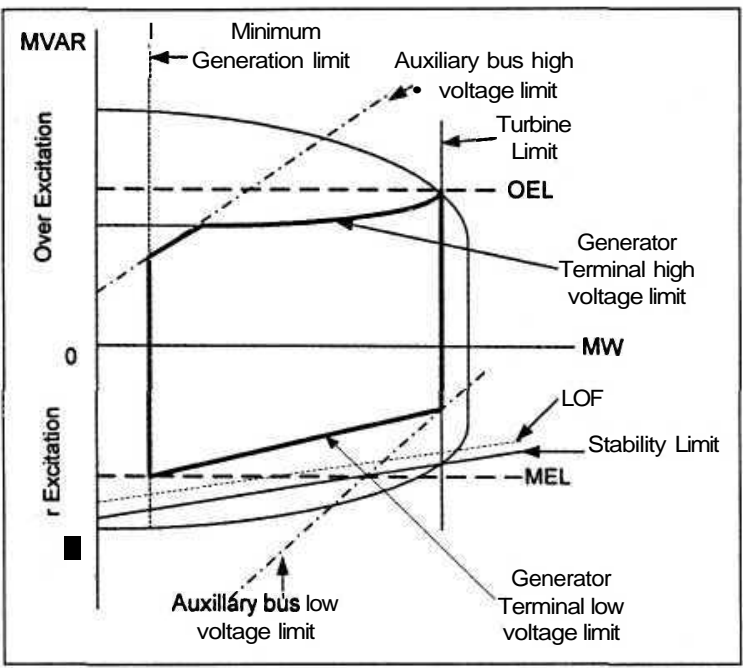

Fig.5: Limitation of reactive power generation capability of a synchronous generator
It is clear that the settings of OEL and UEL are the functions of the winding temperatures. There are advanced adaptive techniques available thereby limiter settings are related with temperature being measured with the sensors embedded at several locations of the windings. Hence, the maximum VAr capability of the machine is possible unlike the fixed limiter setting assuming the machine is always operated at rated conditions. Most of the time utility practice is to provide VAr using capacitor banks and generators are operated at unity power factor keeping the VAr spinning reserve available for emergencies. Therefore machine is normally operated with less winding current than the rated values. In case of an emergency similar machine can be overloaded more than the defined period without reaching the thermal limits if excitation limiter settings are set to vary with the actual winding temperature [9]. IPPs will not consider these techniques to minimize their budget.

The trend of automation of power plants has been introduced another controller to the AVR loop called VAr/pf controller. The objective of $\mathrm{VAr} / \mathrm{pf}$ is to operate the generator at a predetermined power factor and VAr limit irrespective of the network voltages. Normally $\mathrm{VAr} / \mathrm{pf}$ controller is introduced as a minor loop of the AVR. This technique is being widely utilized to control the synchronous motors and later employed in IPPs owned power plants to reduce the operator intervention. Generator incorporating $\mathrm{VAr} / \mathrm{pf}$ controller behaves as voltage following machines and they cannot control the network voltage any more. During transient events $\mathrm{VAr} /$ pf regulators typically do not allow change of machine excitation in response to bring the voltages back to the allowable limits. Hence, utility should introduce necessary restrictions at IPP's generator specifications to avoid incorporating $\mathrm{VAr} / \mathrm{pf}$ controllers to their machines if those generators are expected to be used for controlling the voltage of the network.

Fig.5 shows the operating region limitation of the generator capability curve [6] due to the introduction of various limiters and associated protective devices discussed above. 


\section{Issues related to the step-up transformer}

Special attention should be given to select a suitable step-up transformer for a power plant. Since the generated power is fed to the transmission network through the step up transformer its impedance is a very important parameter. That is because the voltage of the transmission network bus bar of the power plant is dependent on the generator step- up transformer impedance. The step-up transformer impedance is normally $10 \%-15 \%$. Hence, the same amount of voltage drop is noticed at the high voltage bus bar due to the transformer impedance. The generators AVR normally incorporate the drop compensator to overcome the voltage drop due to step-up transformer impedances and thereby generator can control the transmission voltage effectively even though machine is connected to a lower voltage busbar. Thereby maximum benefit of the generator voltage controlling capability can be achieved. Drop compensator parameters are adjusted at the commissioning stage to match up with the step-up transformer used. When system voltage controlling is concerned, drop compensator is very much beneficial and utility should make sure the availability of this facility at IPP generator AVR and keep the correct setting at the commissioning stage.

When generator is not in operation, the auxiliary power is absorbed through the step-up transformer. At similar occasions, especially when the system is being restored after the blackout, step up transformer should be able to provide the permissible voltage to operate the auxiliaries. If the system voltage is not within the allowable limit, it is necessary to use small size generator connected to the auxiliary bus bar to operate auxiliaries and start the generator. However, by incorporating a tap changer to the step-up transformer the auxiliary bus bar voltage can be boosted to the required level when the machine is re-started after the system collapse. Hence, special attention should be given to the tap step and tap settings of the step-up transformer.

At the critical outage situation when the system voltage is decreasing and as a result the stator current limit may be reached for generators with high active power production. Subsequently, the current limiters will bring the stator current to the rated value by reducing the reactive power generation as discussed before. At similar occasions the on load tap changer incorporated to the step up transformer is very beneficial. Operation of the on load tap changer support the machine to generate the maximum possible reactive power without reaching the current limits even at large variations of the system voltage.

Hence on load tap changer with right tap settings defined by considering the transmission network requirement can improve the system reliability. This concept is extensively employed in UK transmission network. In UK, every generator connected to "Super Grid" has a stepup transformer, equipped with an On Load Tap Changer (OLTC). On load tap changers are allowed to respond to the system voltage variations. System operators of some utilities prefer off load tap changers for their generator step-up transformers due to the lack of proper supervisory controlling system and software tools assisting in decision making. However, the tap changer will increase the cost of the transformer. The on load tap changer is $15 \%$ of the total cost of the transformer and also increase the operation and maintenance cost. Therefore IPPs may not support for additional features such as on load tap changers since their prime aim is to minimize the budget.

\section{Transient stability issues}

The generator running in a system should be transiently stable. On the other hands it should be able to remain in synchronism when it is subjected to a sudden disturbance such as a system fault or load variation. Generator rotor angle is the measure of transient stability. Theoretically, the rotor angle should be around $30^{\circ}$ when a machine is operating at the steady state. The rotor angle will fluctuate as a result of the system disturbance, which causes imbalance in the electrical output power of the generator and the mechanical input power to the machine. Then the rotor angle starts swing and if it settles at a new equilibrium state then the system remains in stable. Sometimes larger swings of the rotor angle will cause power fluctuations at the network. At similar occasions system power flow cannot be predicted. Subsequent operation of protection relays isolates the transmissions lines and makes the situation worse. If the rotor angle is continuously increasing the generator become unstable and it should be immediately 
isolated from the network to avoid damage to the generator due to pole slipping. Otherwise generator will be damaged and cascaded tripping of the transmission lines and other connected generators due to power fluctuations may cause a blackout. Always the possible causes for transient instability are deeply considered in system planning, designing and operation stages to eliminate the full or partial system failures.

The main consideration regarding transient stability of a new generator being connected to the network is the time it will take to become unstable when there is a three-phase short circuit fault at the most critical point of the network. This is called the critical clearing time of the unit. The system should be able to isolate the fault before it readers the critical clearing time. Otherwise the generator looses synchronism. Generally the isolation of the network fault at the transmission system within $200 \mathrm{~ms}$ is a reasonable time margin when the recent development in transmission protection equipment are concerned. If the generator becomes unstable before the network fault is isolated it should be disconnected from the system to safeguard both the generator and the network.

The $\mathrm{H}$ constant of the generator and rotor combination is one of the indicators for assessing its transient stability [5]. There are several definitions for $\mathrm{H}$ constant but the ideal definition for this discussion can be given as following. "The $\mathrm{H}$ constant can be defined as half the time it takes the machine to double its speed when full load torque is applied on no load." For instance $\mathrm{H}=\mathrm{l}$ will double its speed in $2 \mathrm{~s}$ if the full load is rejected without change in mechanical torque. There is a rule of thumb in system planning that $\mathrm{H}$ constant should not be less than $1.5 \mathrm{~s}$ for a generator to be connected to the transmission system [10].

The modern trend of IPP is to have a very low $\mathrm{H}$ constant comparing with the typical figures. IPP prefers generators with low $\mathrm{H}$ constant mainly due to the economic advantage of low mass of rotating parts of the generators. For example the recently connected IPPs to the Sri Lankan network have $\mathrm{H}$ constant nearly $1 \mathrm{~s}$ and in a few machines it is less than 1s. Similar machines are generally acceptable for standby generators but not to be operated connected with the national grid. Normally these machines have capacity less than 5MW and a large number of units are operated at one location to provide the desired output. When system reliability is considered these machine reduces the security of the system and cannot remain in synchronism at a network disturbance. The machine reaching to the critical clearing time before the network disturbance is over should be isolated from the network by activating the protection system. Hence, to protect the generator and safeguard the system from a blackout, the generator has to be isolated from the network immediately. Otherwise the system should be operated with a larger spinning reserve available at fast acting units to compensate similar generator disconnections frequently occurring at network disturbances. In addition to this very high rotor angle oscillations can be noticed at low inertia machines even at small system disturbances. To offset the adverse effect of low inertia machines a large investment is necessary for utility to re-install high-speed protection devices and strong transmission interconnections. However, the rotor angle fluctuations of the low inertia machines cannot be completely eliminated. If very low inertia machines are available in a system the best available option is to retrofit a flywheel. Thereby the $\mathrm{H}$ constant can be further increased. Always the utility should decide the minimum possible machine inertia constant and must be more concerned about the related issues on transient stability of the network when the low inertia generator is connected to the network.

Incorporating very fast acting exciters with higher ceiling voltage and response ratio also assists in improving the transient stability of the generator. Static type exciters are more preferred since its time constant is in the range of 0.1s due to the lack of rotating elements. Fast exciter can change the generator terminal voltage rapidly and the system transient stability can be retained by increasing the terminal power output of the generator at severe disturbances. System planners prefer to have fast acting exciters for the machines connected to the transmission system on system security grounds. At extreme operating conditions the thermal capability of exciters will determine the ability of the machine to remain in synchronism. The insulation level of the exciters and the high 
voltage stress on the winding are the limiting factors of the excitation system. Similar to the generators the exciters can also be overloaded for a short period in system disturbances. However, in order to do that additional protection and cooling equipments are necessary. But IPPs will not agree to invest for additional features necessary for improving network reliability and they want to incorporate the excitation system satisfying the minimum operating requirements to match with their financial constraints.

\section{Issues related with the plant protection}

IPPs are more concerned about the protection of their machines and always want them to be isolated from the network when system disturbances are taking place. There are several protection devices incorporated to the generator in order to identify the network disturbances in advance and ensure the subsequent disconnection of the machine from the system. For this purpose under or over voltage/ frequency relays and rate of change of frequency relays (ROCOF), out of step protection relays are commonly used. The utility should be more concerned about the setting of these devices to minimize the unnecessary disconnection of generators even at small system perturbations taking place frequently such as disconnection of small generator at peak load period etc. At similar network disturbances the decrease in voltage and frequency is taking place. The incorrect setting of under/over, voltage/ frequency may disconnect either plant auxiliary or/and the generator from the network Generator tripping further weakens the system by accelerating the frequency falling rate. Apart from that it will disturb the VAr balance of the network. Eventually system may collapse due to transient or voltage instability. At similar instances proper coordination of above discussed plant protecting relays with network disturbance recovery measures such as load shedding schemes or application of secondary spinning reserve etc. is extremely important [11]

Hence, the machine should be designed to be in operation and provide the maximum possible power within the voltage and frequency variation ranges defined in the grid code or operational procedures. According to the operational practice under system contingencies

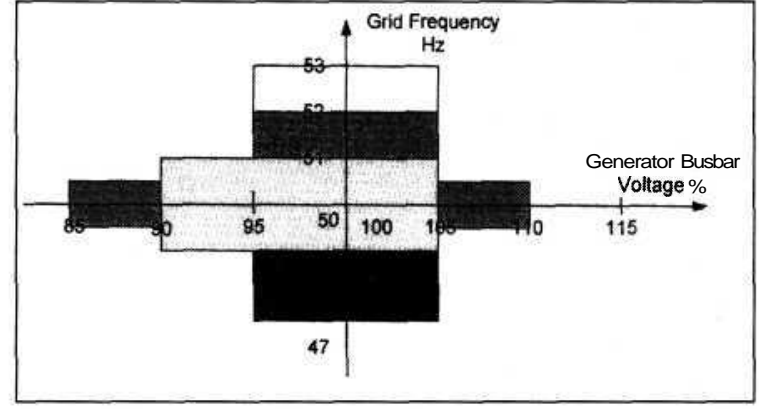

Fig.6: The acceptable operating limits recommended for thermal units of Sri Lankan power system.

the transmission voltage variation should be in the range of $-10 \%$ and $5 \%$ and the generators and auxiliaries should be able to operate continuously providing the maximum possible power if the system frequency is within $50.5 \mathrm{~Hz}$ to $49 \mathrm{~Hz}$. At voltages and frequencies of outside the normal operating range but within the maximum allowable limits the total power can not be expected from the machine but depending on the plant type a fraction of the power should be available for at least small period such as 30 minutes. The maximum allowable voltage is a transmission system design parameter and the minimum allowable voltage is determined by considering the behavior of the plant auxiliaries. The minimum and maximum operating frequency will be dependent on the prime mover characteristics of the plant. These limits should be clearly mentioned at the utility generator specification. Fig. 6 shows the acceptable operating limits for thermal units concerning probable tolerances of frequency and voltage fluctuations at normal and transient states of the Sri Lankan power system. Special attention should be made at the commissioning stage to verify the settings of under/over frequency and voltage relays of plant auxiliaries.

IPP plants are normally incorporated with ROCOF relays to safeguard machines from fast drop in frequency. ROCOF relay settings of the generating plants should be determined by considering realistic critical outages of the entire transmission system. The maximum impact on the system frequency takes place due to the sudden tripping of a large machine or a heavily loaded line when the system has very small load. At similar occasions the fast drop in frequency results in operating ROCOF relays and disconnecting machines thereby further increasing the frequency falling rate. ROCOF 
relay of IPP plants is set just by considering as a plant protection mean without getting the views of system operators. Generally, relay is set to activate even at small frequency disturbances though the system frequency can be restored within few seconds. Hence, it is correct to assume that majority of IPP machines disconnects from the system even at a small disturbance. Therefore system should be operated with higher spinning reserve considering the possible generation deficit due to the disconnection of IPPs at critical disturbances. Therefore several fast acting generating units have to be operated below the rated operating load to maintain spinning reserve irrespective of the economic operation principles to safeguard the system from blackouts. The utility planners should fully understand the similar hidden expenditure to be borne by the utility when an IPP plant is operated at the system and the technical specification should be thoroughly revised to include necessary provisions at least to minimize their adverse effects.

\section{Power plant operational issues}

The utility owned plant operators are fully trained about the power plant features and transmission network behavior at the emergency situations. They have been well trained on manual operation of the generator at system disturbances and support to the network operator to overcome possible system failures. For example if machine excitation has reached to the maximum level and alarms are ringing prior to disconnection of the generator from the system the well trained operator can takeover the manual operation until another machine is started somewhere else in the network to replace the active and reactive power provided by the faulty generator. Otherwise the sudden disconnection may overload other transmission facilities and load shedding may be necessary to restore the system frequency. Instead of sudden automatic disconnection of the machine due to the minor internal faults such as ground fault at field winding, out of service AVR, excitation limited by slip ring arcing, load limited by a fuel supply problem etc., the utility plant operator is capable of manually operating the machine for a reasonable time period sufficient for network operator to reschedule the generation. The risk of damage to the plant is accepted for the greater value of the reduced risk of having a blackout. However, IPPs always prefer to have automatic control for their machines to minimize the operator intervention and will not sacrifice lifetime of their machines for the benefit of the network.

\section{Conclusions:}

The following conclusions can be made based on the above discussion.

1. IPPs main objective is to get a good return for their investment and as a consequence the utility has to sacrifice the system reliability.

2. The generator parameters and the expected behavior of the plant at operational voltage and frequency ranges should be clearly indicated at the specifications given to IPPs by the utility.

3. Engineers of the utility should actively participate for power plant commissioning and should come to compromise with IPPs for protection settings and controller tuning favourable to the system operations.

4. Many problems are due to the non-payment for IPPs for their reactive power generation. Utility should think about a method to encourage IPPs for reactive power generation by introducing payment scheme for VArh generated when generator power factor is kept below 0.9 or some reasonable value.

5. Detailed power system analysis should be carried out under the feasibility study to identify possible problems in advance and to suggest remedial measures.

6. Power plant operators should be well trained to face for generator operation at network disturbances and at least IPPs should be persuaded to ensure the operation of their machines at some previously agreed upon problematic conditions. 


\section{References}

1. Statistical Digest of CEB-2006

2. Statistical Digest of CEB-2001

3. Power System Policy Directions by Ministry of Irrigation and Power in 1998

4. Power System Operation by Robert H. Miller and james H. malinowski

5. Power System Engineering by Nagrath and kothari

6. NERC (North American Electric Reliability Council) operating Manual

7. M.M. Adibi and D.P. Milanicz " Reactive Capability Limitation of Synchronous machines", IEEE Transactions on Power Systems, Vol.9, No.1, pp. 29-40, February 1984.

8. J.R. Ribeiro, "Minimum Excitation Limiter effects on Generator Response to system Disturbances", IEEE transactions on energy Conversion, Vol.6, No.1,pp.29-38, March 1991.

9. C.W. Taylor, "Survey of effective and practical solutions for longer term Voltage Stability", International Journal of Electrical Power and Energy Systems, Vol.15, No.4, pp. 217-220, August 1993.

10. H.J. Langley “ Inertia of Small Generators" IEE Power Engineering Journal, pp. 196,Vol. 14, Number 4

11. JL.H. Fink and K. Carlsen “ Operating under stress and Strain" IEEE Spectrum, pp.48-53, March 1978 